\title{
Prostate Stereotactic Body Radiation Therapy With Halcyon 2.0: Treatment Plans Comparison Based on RTOG 0938 Protocol
}

Yücel Altundal ${ }^{1}$, Fulya Cifter ${ }^{2}$, Guangwei Mu ${ }^{1}$, James Lee ${ }^{3}$, Elisa J. Wu ${ }^{3}$, Vincent Yeung ${ }^{3}$, Alan Katz

1. Medical Physics, Flushing Radiation Oncology Services, Flushing, USA 2. Medical Physics, Precess Medical, Short Hills, USA 3. Radiation Oncology, Flushing Radiation Oncology Services, Flushing, USA 4. Radiation Oncology, St. Francis Hospital, Roslyn, USA

Corresponding author: Yücel Altundal, altundal@gmail.com

\section{Abstract}

\section{Purpose}

The aim of this study is to investigate the feasibility of prostate stereotactic body radiation therapy treatment with a newly developed Varian Halcyon ${ }^{\mathrm{TM}} 2.0$ machine by comparing radiotherapy plans with previously delivered CyberKnife G4 plans created with the previous version of CyberKnife Treatment Planning System Multiplan 4.6.1.

\section{Methods}

Fifteen previously treated prostate stereotactic body radiation therapy treatment CyberKnife plans were replanned retrospectively according to the Radiation Therapy Oncology Group 0938 protocol on a Halcyon ${ }^{\mathrm{TM}}$ 2.0 machine with a prescription of $3625 \mathrm{cGy}$ in five fractions.

\section{Results}

All re-plans on a Halcyon ${ }^{\mathrm{TM}} 2.0$ were able to meet the Radiation Therapy Oncology Group 0938 protocol goals and constraints. The re-plans decreased the maximum dose to skin and urethra, mean doses to the bladder and rectum, and also improve the conformity index and the Planning Target Volume coverage. However, D1cc to the rectum, D1cc and D10\% to the bladder increased with no statistically significant differences $(\mathrm{p}>0.05)$ with the re-plans.

\section{Conclusion}

The Halcyon ${ }^{\mathrm{TM}} 2.0$ can generate stereotactic body radiation therapy treatment prostate plans created based on the Radiation Therapy Oncology Group 0938 protocol by delivering adequate coverage to the target while sparing healthy tissues.

Review began 10/31/2020 Review ended 11/11/2020 Published 11/23/2020

๑) Copyright 2020

Altundal et al. This is an open access article distributed under the terms of the Creative Commons Attribution License CC-BY 4.0, which permits unrestricted use, distribution, and reproduction in any medium, provided the original author and source are credited.
Categories: Medical Physics, Radiation Oncology, Healthcare Technology

Keywords: radiotherapy, sbrt, halcyon, rtog 0938, cyberknife

\section{Introduction}

Hypo-fractionated stereotactic body radiation therapy (SBRT) is a method to deliver large and precise doses of radiation externally to an extracranial target within the body in a few fractions [1]. Administration of highly conformal radiation with this technique to small, well defined, and well-targeted tumors spares the surrounding healthy tissue by rapid dose fall-off. For prostate cancer patients, the conventional external beam therapy lasts about eight to nine weeks, up to about 80 Gy [2]. SBRT for prostate treatment uses higher daily doses in fewer fractions, which is radiobiologically beneficial due to the low alpha/beta ratio of prostate cancer [3].

The long-term good outcomes with minimal toxicity of prostate cancer SBRT treatment using the CyberKnife System (Accuray Incorporated, Sunnyvale, CA, USA) has been discussed in several papers [4-9]. This system uses an image-guided linear accelerator mounted on a robotic arm to deliver external beam radiation [10]. The real-time image guidance with five or six-degrees-of-freedom couch provides precise delivery of high radiation doses to the target by tracking fiducials implanted in the prostate [11,12], and correcting the position errors in near real time by manipulating the treatment head using the highly precise industrial robot attached to the treatment head.

Meanwhile, a newly developed closed system linear accelerator (LINAC) with a 100-cm diameter bore size, Halcyon $^{\mathrm{TM}}$ (Varian Medical System, Palo Alto, CA, USA), was introduced to simplify the treatment workflow 
and speed up the delivery of the radiation. Halcyon ${ }^{\mathrm{TM}} 2.0$ with a $\mathrm{kV}$ cone-beam computed tomography (CBCT) delivers a single 6MV flattening filter-free (FFF) beam with a double stack multi-leaf collimator (MLC), SX2. The width of SX2 leaves is $1 \mathrm{~cm}$ at the isocenter with a $0.5-\mathrm{cm}$ offset at the isocenter to minimize the leakage between the proximal and distal leaves. This double stack MLC design renders an effective leaf resolution of $5 \mathrm{~mm}$ at the isocenter. The SX2 collimator allows both the proximal and distal leaves to modulate the beam. The increased gantry speed is $4 \mathrm{rpm}$, the maximum leaf speed is $5 \mathrm{~cm} / \mathrm{s}$ with a dose rate of $800 \mathrm{MU} / \mathrm{min}$ at dmax of $1.3 \mathrm{~cm}$ for a $100-\mathrm{cm}$ SSD setup [13,14].

This study aims to investigate the feasibility of prostate SBRT treatment with a Halcyon ${ }^{\mathrm{TM}} 2.0$ linac. For this purpose, 15 SBRT prostate plans previously delivered at a CyberKnife (CK) were compared with plans recreated for a Halcyon ${ }^{\mathrm{TM}} 2.0$. The CyberKnife plans were generated based on the Radiation Therapy Oncology Group (RTOG) 0938 protocol prescription, goals, and constraints [15]. The plans were compared based on the RTOG 0938 protocol. In addition to those goals and constraints, mean doses of planning target volume (PTV), rectum, and bladder; minimum dose to prostate; PTV and prostate dose coverages; conformity index (CI), Paddick conformity index (PCI) and Homogeneity index (HI) were also compared $[16,17]$.

\section{Materials And Methods \\ Patient selection}

Fifteen prostate cancer patients previously treated at the Flushing Radiation Oncology Center (FROS) between August 2019 and May 2020 with a total prescription of 3625 cGy were selected randomly. These patients were treated with a CyberKnife G4 unit in five fractions according to the RTOG 0938 protocol.

\section{Imaging, delineation, and SBRT treatment plan requirements}

Computed tomography (CT) simulations were performed with a 1-mm scan slice thickness in a feet-first supine position and then fused with $2 \mathrm{~mm}$ T2 magnetic resonance (MR) images. Fusions were performed by matching four fiducials implanted into the prostate. The corresponding physicians contoured prostate (clinical target volume), rectum, bladder, urethra, penile bulb, and bowels. Planning target volumes were created by extending the clinical target volume (CTV) $3 \mathrm{~mm}$ posteriorly, and $5 \mathrm{~mm}$ in all other directions. The planner contoured femoral heads and $15 \mathrm{~mm}$ thick skin.

\section{Treatment planning}

A CyberKnife G4 Image-Guided Robotic Stereotactic Radiosurgery System unit with a variable IRIS was used to treat those patients. The IRIS circular collimator delivers cone-shaped beams with a diameter of 5 to 60 $\mathrm{mm}$ nominal size at a SAD of $80 \mathrm{~cm}$. Multiplan 4.6.1 TPS with Ray-Tracing calculation method and sequential optimization algorithm was used to generate plans. All plans were delivered with the IRIS collimator. The prescription dose was prescribed to 78-81\% isodose lines to get coverage of $93.99-95.44 \%$ of the PTV volume. The minimum and maximum MU sets were 25 and 770 per beam, respectively. A computercontrolled five degrees of freedom (DoF) treatment couch was used to position the patient, the yaw rotational correction being the left out. The rotational (yaw) corrections were corrected manually in the room by therapists before start of the treatment delivery. The gold fiducials implanted inside the prostate were used for target localization and tracked during the treatments with two orthogonal kilo-voltage X-ray and detector systems by imaging in about every 30-40 seconds. The linac delivered 6MV flattening filter-free (FFF) Photon Energy with a maximum dose rate of $1000 \mathrm{MU} / \mathrm{min}$.

The Halcyon ${ }^{\mathrm{TM}}$ plans were generated with the Eclipse TPS version of 15.6.06. The inverse planning technique with the photon optimizer (PO) version of 15.6 .06 was used, and dose calculations were performed with the analytical anisotropic algorithm (AAA) version of 15.6.06. Three or four full arcs with fine resolution $(1.25 \mathrm{~mm}$ ) were used to generate coplanar volumetric modulated arc therapy (VMAT) plans. Eight of the plans were generated with three full arcs with automatically generated 285, 345, and 45 degrees of collimator angles. Four full arcs with automatically generated 281, 326, 11, and 56 degrees of collimator angles were used for the rest of the plans. In addition to RTOG protocol (Table 1), the planner aimed to have at least $99 \%$ of the volume of the prostate (CTV) to get 100\% of the prescription dose ( $3625 \mathrm{cGy}$ ) and minimize the doses to the healthy tissues as much as possible. Once plans were satisfied with the desired coverages and constraints, no more adjustments were made.

\section{The RTOG 0938}

The RTOG 0938 goals and constraints given in Table 1 of CK and Halcyon plans were compared [15]. 


\section{Cureus}

\begin{tabular}{|c|c|c|c|}
\hline Organ & Volume & $\begin{array}{l}\text { Dosimetry parameters for } 5 \text { fraction arm and } \\
\text { with all delivery devices except CyberKnife }\end{array}$ & $\begin{array}{l}\text { Dosimetry parameters for } 5 \text { fraction } \\
\text { arm and treatment with CyberKnife }\end{array}$ \\
\hline \multirow{3}{*}{ PTV } & Max point dose & $\leq 38.78$ Gy, $107 \%$ of prescription & $\leq 43.5 \mathrm{~Gy}, 120 \%$ of prescription \\
\hline & $\begin{array}{l}\text { Min dose } \\
\text { received by } 95 \% \\
\text { of PTV }\end{array}$ & $\geq 36.25$ Gy, $100 \%$ of prescription & SAME \\
\hline & $\begin{array}{l}\text { MIn dose } \\
\text { received by PTV }\end{array}$ & $\geq 34.4$ Gy, $95 \%$ of prescription & SAME \\
\hline \multirow{5}{*}{ Rectum } & $\begin{array}{l}\text { Max point dose } \\
\text { (1cc) }\end{array}$ & $\leq 38.06$ Gy, $105 \%$ of prescription & SAME \\
\hline & Less than $3 \mathrm{cc}$ & $<34.4$ Gy, $95 \%$ of prescription & SAME \\
\hline & $10 \%$ rectum & $\leq 32.625$ Gy, $90 \%$ of prescription & SAME \\
\hline & $20 \%$ rectum & $\leq 29$ Gy, $80 \%$ of prescription & SAME \\
\hline & $50 \%$ rectum & $\leq 18.125$ Gy, $50 \%$ of prescription & SAME \\
\hline \multirow{3}{*}{ Bladder } & $\begin{array}{l}\text { Max point dose } \\
(1 \mathrm{cc})\end{array}$ & $\leq 38.06$ Gy, $105 \%$ of prescription & SAME \\
\hline & $10 \%$ bladder & $\leq 32.625$ Gy, $90 \%$ of prescription & SAME \\
\hline & $50 \%$ bladder & $\leq 18.125$ Gy, $50 \%$ of prescription & SAME \\
\hline \multirow{2}{*}{$\begin{array}{l}\text { Penile Bulb } \\
\text { (recommended) }\end{array}$} & Max point dose & No more than $100 \%$ of prescription & SAME \\
\hline & Less than 3cc & 20 Gy, $54 \%$ of prescription & SAME \\
\hline \multirow{2}{*}{$\begin{array}{l}\text { Fem Heads_L } \\
\text { (recommended) }\end{array}$} & Max point dose & $30 \mathrm{~Gy}, 81 \%$ of prescription & SAME \\
\hline & Less than $10 \mathrm{cc}$ & 20 Gy, $54 \%$ of prescription & SAME \\
\hline \multirow{2}{*}{$\begin{array}{l}\text { Fem Heads_R } \\
\text { (recommended) }\end{array}$} & Max point dose & 30 Gy, $81 \%$ of prescription & SAME \\
\hline & Less than $10 \mathrm{cc}$ & 20 Gy, $54 \%$ of prescription & SAME \\
\hline \multirow{2}{*}{$\begin{array}{l}\text { Skin } \\
\text { (recommended) }\end{array}$} & Max point dose & 30 Gy, $81 \%$ of prescription & SAME \\
\hline & Less than $10 \mathrm{cc}$ & 20 Gy, $54 \%$ of prescription & SAME \\
\hline Urethra dose & Max point dose & $\leq 38.78$ Gy, $107 \%$ of prescription & SAME \\
\hline
\end{tabular}

TABLE 1: The RTOG 0938 goals and constraints.

\section{Other dosimetric parameters}

In addition to RTOG 0938 goals and constraints, PTV mean dose, the percentages of PTV and CTV volumes that receive prescription dose (coverage), the minimum prostate dose, rectum mean dose, bladder mean dose, CI, PCI and HI of the CK and Halcyon plans were compared. The CI, PCI and HI are defined as follows:

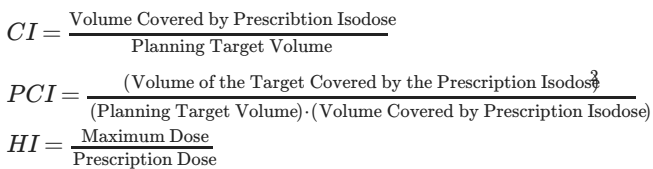

Conformity index given by the first equation was defined by RTOG radiosurgery guidelines to evaluate how well a target is covered by the volume of the prescription isodose lines [18]. However, CI does not take into account the shape and the location of the prescription isodose line volume. To account this Paddick conformity index (second equation) was proposed by Paddick [16] to evaluate the degree of overlapping of the target volume and the prescription isodose line volume. The values for both the CI and PCI close to 1.0 are considered more conformal. On the other hand, the homogeneity index is useful to evaluate the homogeneity of dose distribution within the target. A value of 1.0 indicates a perfect homogeneous plan. 


\section{Cureus}

\section{Statistical analysis}

The small sample size nonparametric Wilcoxon signed-rank test was used to analyze all the data with a pvalue less than 0.05 considered to be statistically significant (IBM ${ }^{\circledR}$ SPSS ${ }^{\circledR}$ Statistics Subscription, IBM Corporation, Armonk, NY).

\section{Plan quality assurance (QA)}

The pre-treatment patient plan quality assurance (QA) of each plan was performed by using Varian Portal Dosimetry (PD) with a $2 \% / 2 \mathrm{~mm}$ gamma index passing metric and a $10 \%$ threshold.

\section{Results}

RTOG 0938

The average values of the RTOG 0938 goals and constraints with standard deviations and p-values of 15 plans are given in Table 2. All the Halcyon VMAT plans can satisfy the goals and constraints of the RTOG 0938 protocol hence the average values. However, the averaged CK plans fail to satisfy any of the PTV goals and the urethra constraint. There are statistically significant differences in the goals and the constraints of the protocol between the CK and Halcyon plans except for D1cc, D3cc, and D50\% of the rectum, D1cc and D10\% of the bladder constraints. 


\section{Cureus}

\begin{tabular}{|c|c|c|c|c|c|c|}
\hline \multirow[b]{2}{*}{ Organ } & \multirow[b]{2}{*}{ Volume } & \multicolumn{2}{|l|}{ CK } & \multicolumn{2}{|l|}{ Halcyon } & \multirow[b]{2}{*}{$\mathrm{p}$-value } \\
\hline & & Average (cGy) & STD (cGy) & Average (cGy) & STD (cGy) & \\
\hline \multirow[t]{3}{*}{ Prostate (PTV) } & Max point dose & 4547 & 46 & 3843 & 22 & $<0.001$ \\
\hline & Min dose received by $95 \%$ of PTV & 3618 & 12 & 3635 & 13 & 0.006 \\
\hline & Min dose received by PTV & 2896 & 186 & 3479 & 30 & $<0.001$ \\
\hline \multirow[t]{5}{*}{ Rectum } & Max point dose (1cc) & 3566 & 74 & 3609 & 66 & 0.061 \\
\hline & Less than $3 \mathrm{cc}$ & 3298 & 94 & 3273 & 128 & 0.495 \\
\hline & $10 \%$ rectum & 3118 & 131 & 3041 & 145 & 0.017 \\
\hline & $20 \%$ rectum & 2595 & 210 & 2419 & 141 & 0.036 \\
\hline & $50 \%$ rectum & 1527 & 228 & 1416 & 216 & 0.100 \\
\hline \multirow[t]{3}{*}{ Bladder } & Max point dose (1cc) & 3670 & 145 & 3695 & 42 & 0.670 \\
\hline & $10 \%$ bladder & 2716 & 463 & 2789 & 416 & 0.334 \\
\hline & $50 \%$ bladder & $121 /$ & 421 & 909 & 502 & 0.001 \\
\hline \multirow[t]{2}{*}{ Penile Bulb } & Max point dose & 2832 & 616 & 2522 & 802 & 0.004 \\
\hline & Less than $3 \mathrm{cc}$ & 473 & 578 & 214 & 253 & N/A \\
\hline \multirow{2}{*}{ Fem Heads_L } & Max point dose & 938 & 285 & 1169 & 238 & 0.005 \\
\hline & Less than $10 \mathrm{cc}$ & 549 & 219 & 829 & 208 & 0.003 \\
\hline \multirow[t]{2}{*}{ Fem Heads_R } & Max point dose & 1482 & 248 & 1212 & 233 & 0.020 \\
\hline & Less than $10 \mathrm{cc}$ & 1105 & 214 & 890 & 216 & 0.017 \\
\hline \multirow[t]{2}{*}{ Skin } & Max point dose & 1849 & 45 & 1518 & 138 & $<0.001$ \\
\hline & Less than $10 \mathrm{cc}$ & 1452 & 105 & 1205 & 117 & $<0.001$ \\
\hline Urethra dose & Max point dose & 4158 & 109 & 3793 & 22 & $<0.001$ \\
\hline & evel Is 0.05 . & & & & & \\
\hline
\end{tabular}

TABLE 2: Average values, standard deviations and p-values of CyberKnife and Halcyon plans.

PTV: Planning target volume

\section{Other dosimetric parameters}

Figure 1 shows the average values of coverages of PTV and CTV; minimum dose to CTV; mean doses of PTV, rectum, and bladder; conformity index, Paddick conformity index and homogeneity index with one standard deviation, maximum and minimum values of Halcyon and $\mathrm{CK}$ plans. There are no statistically significant differences only between the prostate dose coverage and prostate minimum dose among those parameters. 


\section{Cureus}

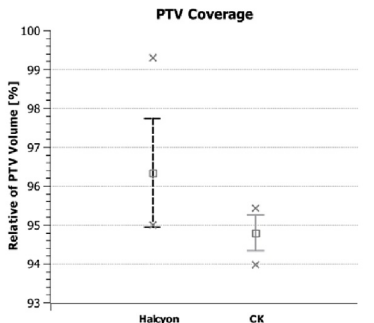

(a) $\quad p=0.006$

PTV Mean Dose

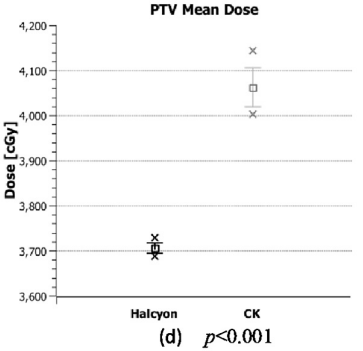

Conformity Index

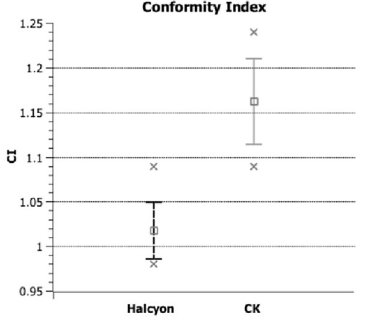

(g) $p<0.001$

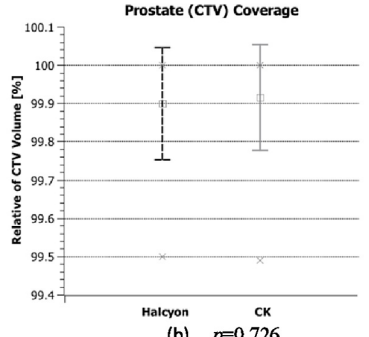

(b) $p=0.726$

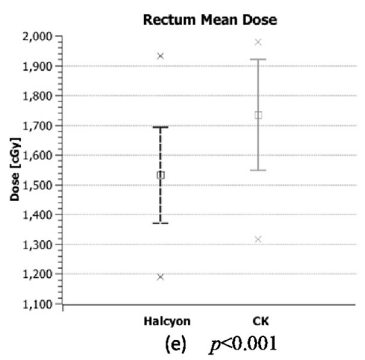

Paddick Conformity Index

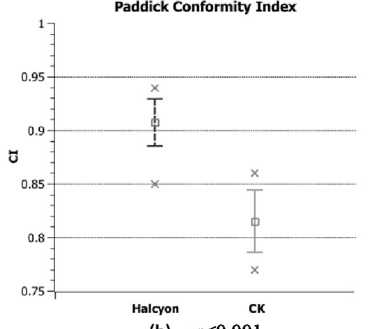

(h) $p<0.001$
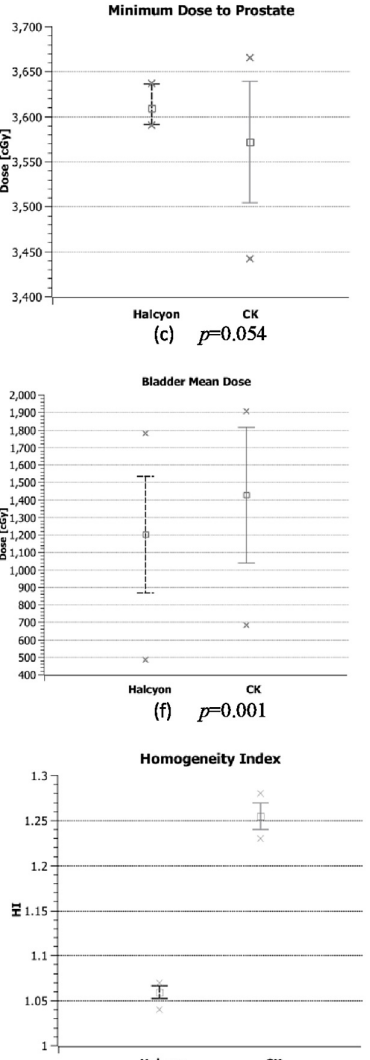

(i) $p<0.001$

\section{FIGURE 1: Average values of dosimetric parameters.}

Average values, one standard deviation (bars), maximum and minimum (x) values of (a) Planning Target Volume (PTV) coverage, (b) CTV coverage, (c) Minimum point dose to Clinical Target Volume (CTV), (d) Planning Target Volume (PTV) mean dose, (e) Rectum mean dose, (f) Bladder mean dose, (g) Conformity Index, (h) Paddick Conformity Index and (i) Homogeneity Index of CyberKnife (CK) and Halcyon plans.

The prescription dose coverage (volume getting 100\% of the prescription dose) of the PTV and CTV are 96.34 $\pm 0.36 \%$ (mean \pm standard error) and $99.90 \pm 0.04 \%$, respectively; the average mean doses of the PTV, bladder and rectum are $3706 \pm 3 \mathrm{cGy}, 1203 \pm 86 \mathrm{cGy}$ and $1533 \pm 42 \mathrm{cGy}$, respectively; and the CI, PCI and HI are $1.02 \pm$ $0.01,0.91 \pm 0.01,1.06 \pm 0.01$, respectively, for the Halcyon VMAT plans.

\section{Monitor unit and delivery time for Halcyon plans}

Figure $2 a$ and Figure $2 b$ show the average values of monitor units (MU), and QA beam-on time with one standard deviation, maximum and minimum values for three and four arc VMAT Halcyon plans, respectively. For 3-Arc plans, the average total monitor unit and QA beam-on time are $3093.9 \pm 134.9 \mathrm{MU}$ and $4.12 \pm 0.18 \mathrm{~min}$, respectively, for one fraction. For 4-Arc plans, the average total monitor unit and QA beam-on time are $3143.9 \pm 88.5 \mathrm{MU}$ and $4.19 \pm 0.12 \mathrm{~min}$, respectively. The monitor unit and beam-on time per beam for 3-Arc plans are $1031.3 \pm 31.2 \mathrm{MU}$ and $1.37 \pm 0.04 \mathrm{~min}$, respectively. For 4-Arc plans, the monitor unit and beam-on time per beam are $786.0 \pm 12.3 \mathrm{MU}$ and $1.05 \pm 0.02 \mathrm{~min}$, respectively. Owing to the high number of small segments in CK, the high number of MU's was a limitation to compare (i.e. 36,333 $\pm 455 \mathrm{MU}$ vs $3143.9 \pm 88.5 \mathrm{MU})$. 


\section{Cureus}

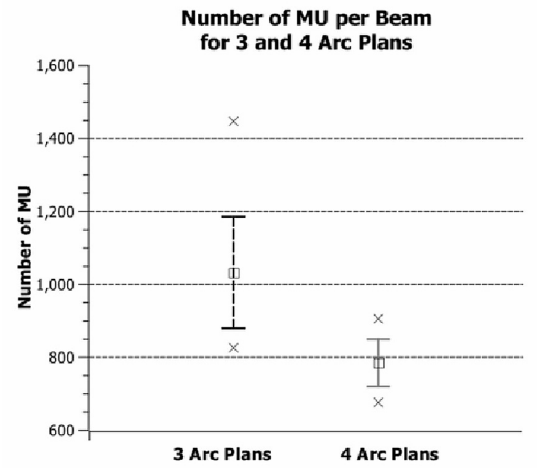

(a)

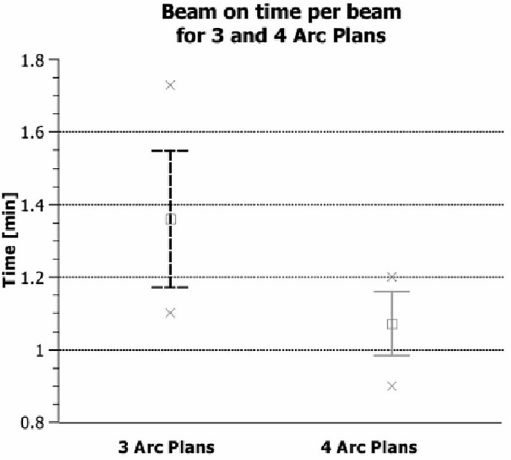

(b)

\section{FIGURE 2: Monitor unit and delivery time for Halcyon plans.}

Average values, one standard deviation, maximum and minimum values of (a) MU per beam and (b) beam-on time per beam for three and four arc Halcyon plans.

\section{Plan QAs}

The QA pass rate of each beam is $99.75 \pm 0.08$ with a $2 \% / 2 \mathrm{~mm}$ gamma index and a $10 \%$ threshold.

\section{Discussion}

\section{Dosimetric comparison of CK and Halcyon plans}

The averaged dose volume histograms (DVH) for the targets and critical structures of the CyberKnife and Halcyon plans are given in Figure 3. The Halcyon ${ }^{\mathrm{TM}} 2.0$ is capable of generating plans which satisfy the plan criteria of RTOG 0938 protocol. However, plans have significant dose differences with CyberKnife plans in high dose regions for the targets and low dose regions for the critical organs, except urethra and left femoral head. Those differences in the DVHs are mainly because of the different designs of the two systems.

Halcyon ${ }^{\mathrm{TM}} 2.0$ delivers isocentric, coplanar and MLC modulated arc beams; on the other hand, the CyberKnife G4 system delivers non-isocentric, non-coplanar and cone-shaped beams. Several studies have shown that isocentric arc plans modulated with multi-leaf collimators provide excellent dose conformity and coverage of the target [19-22]. VMAT also provides homogeneous plan with high dose gradient [23]. Similar to those studies, Halcyon VMAT plans generated cooler and more conformal plans compared to CK system. There are statistically significant improvements $(\mathrm{p}<0.05)$ on the conformity, Paddick conformity and homogeneity indexes (Figure 1 1 -1i). The maximum dose to skin and 50\% isodose line fall-off are better with VMAT Halcyon plans as well (Figure 4 and Figure 5). Also, there are significant differences between high dose parts of PTV, CTV, urethra and skin DVHs and mean doses of rectum and bladder. Since all these values decreased significantly at VMAT plans, increasing the prescription dose (i.e. $3750 \mathrm{cGy}$ ) is an option to increase the mean PTV dose with Halcyon ${ }^{\mathrm{TM}} 2.0$. 


\section{Cureus}

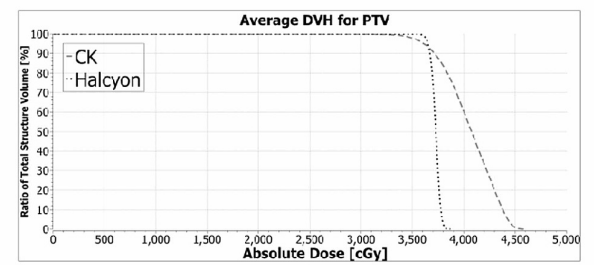

(a)

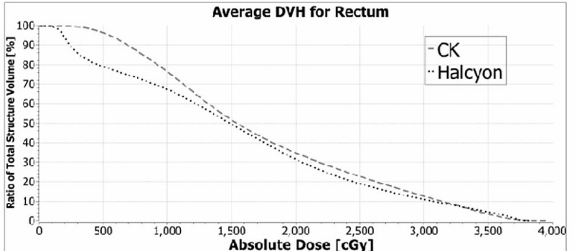

(c)

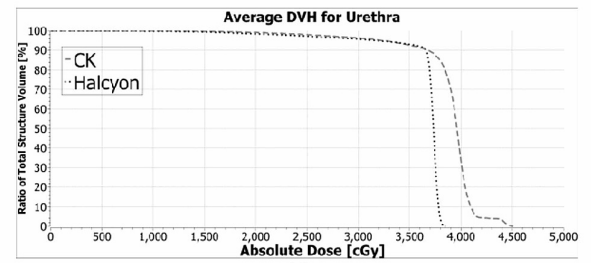

(e)

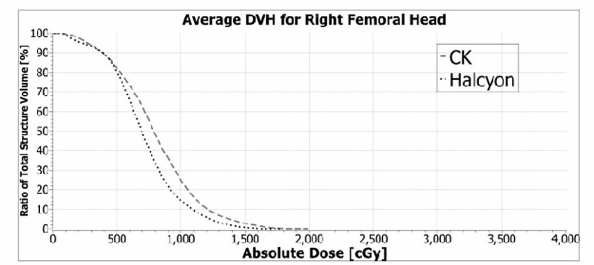

(g)

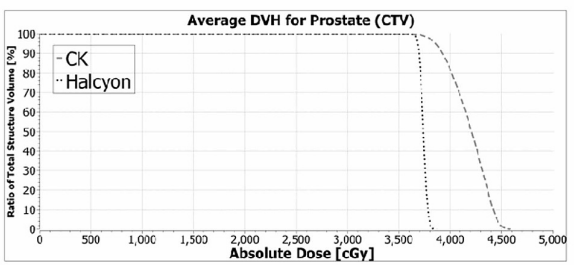

(b)

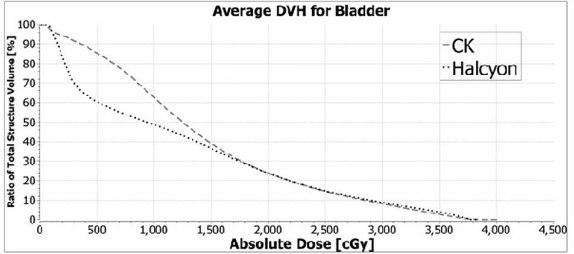

(d)

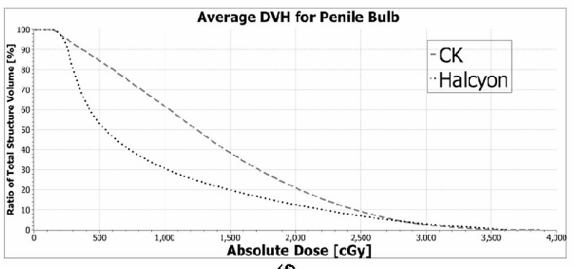

(f)

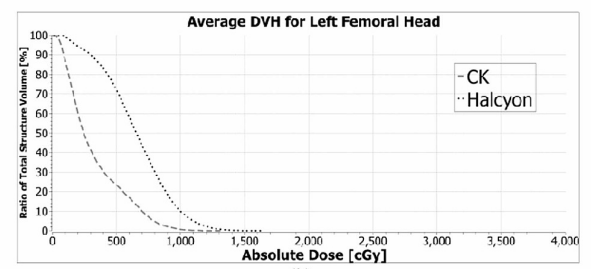

(h)

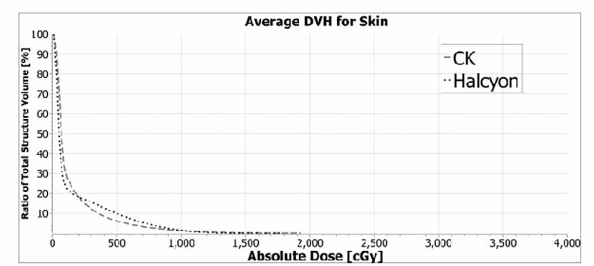

(i)

FIGURE 3: Average dose volume histograms of CK and Halcyon plans. 


\section{Cureus}

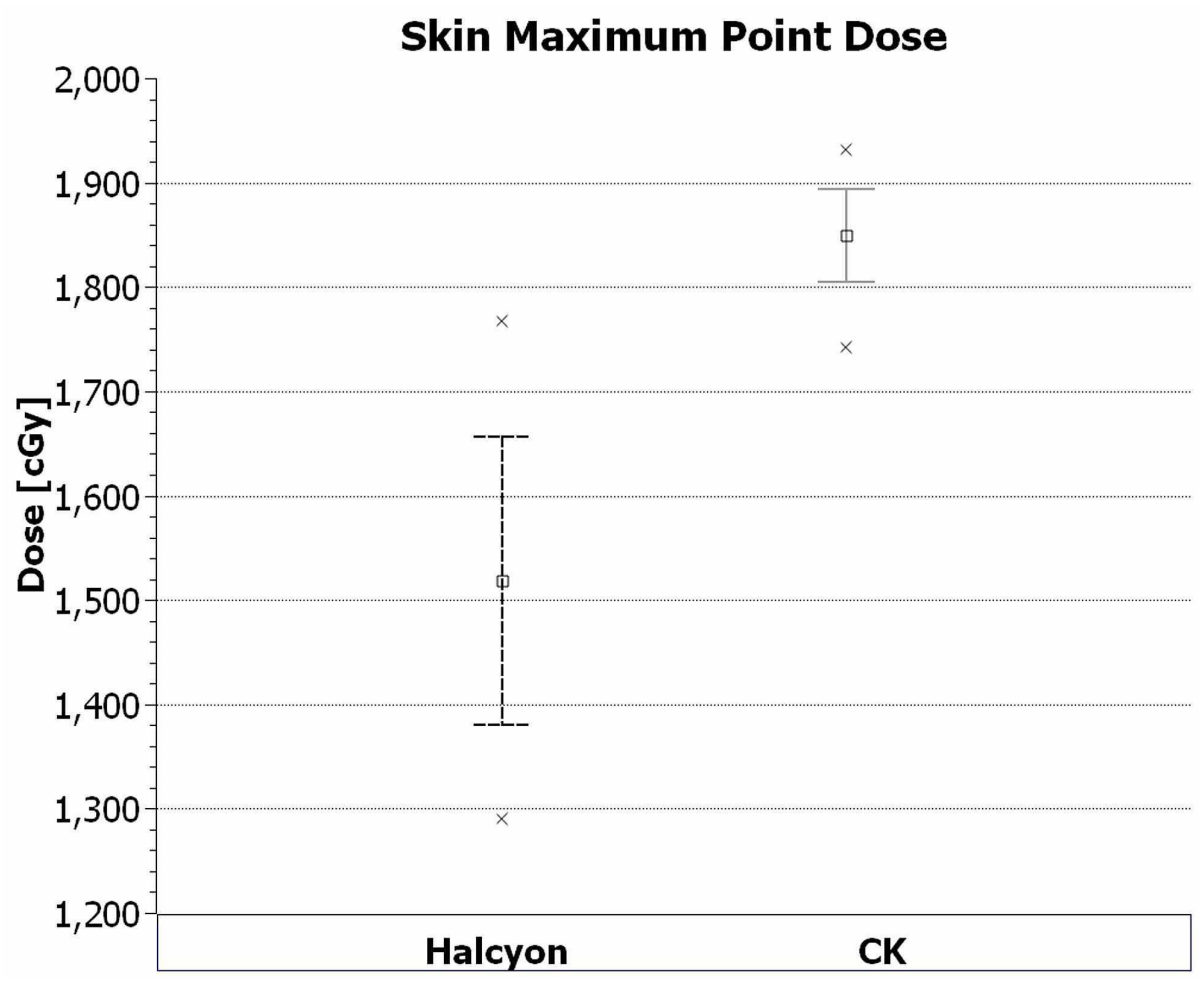

FIGURE 4: Average maximum dose to skin.

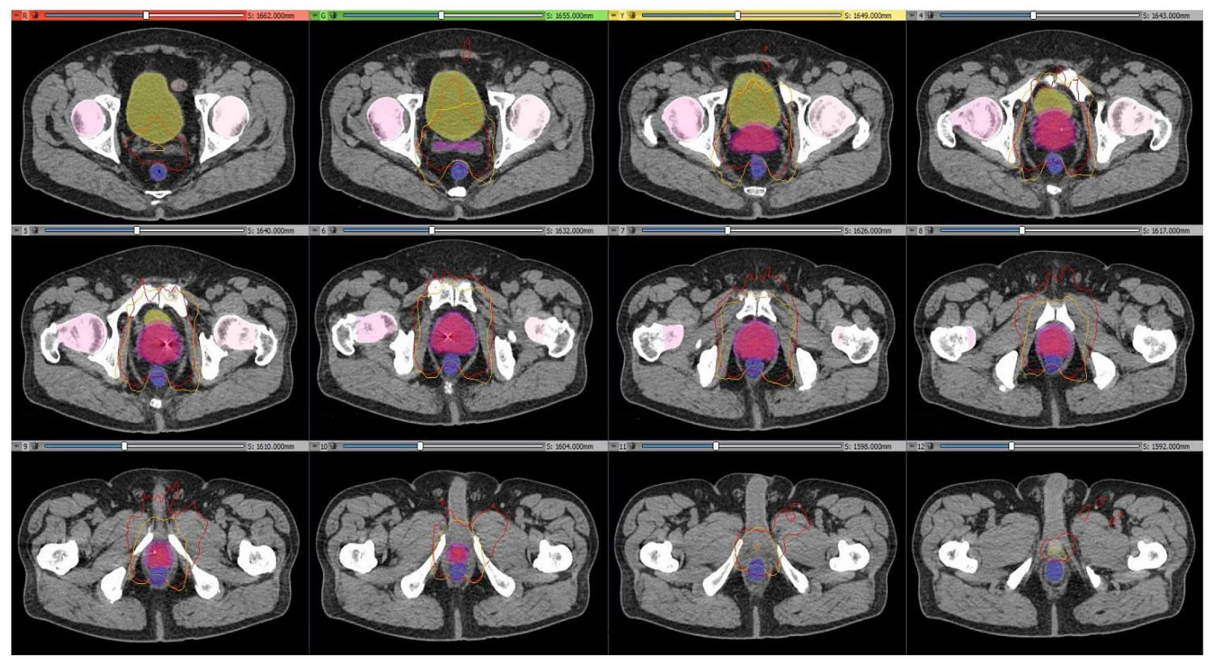

FIGURE 5: An example of $\mathbf{5 0 \%}$ isodose lines.

An example of a patient axial images: $50 \%$ isodose lines of Halcyon (orange lines) and CK (red lines) plans. Images, structures and isodose lines were generated with 3D Slicer 4.10.2 [24].

Regarding the organs at risk, dose volume histograms show that Halcyon plans improved almost all the critical organ constraints except the left femoral head. The left femoral head dose from CyberKnife plans is lower because of the robotic design of the machine. The CK non-coplanar plans are delivering less dose through the left side of the feet first supine positioned patients. The average values of Halcyon plans are higher for D1cc of the rectum, D1cc and D10\% of the bladder constraints. However, there are no statistically significant differences among those values between CK and Halcyon plans $(\mathrm{p}>0.05)$. Also, bladder and rectum mean doses decreased significantly with Halcyon 2.0 plans (Figure 1e and Figure 1f).

In addition to DVH differences, Halcyon ${ }^{\mathrm{TM}} 2.0$ fast arc delivery generated highly conformal plans (Figure $1 g$ and Figure $1 h$ ) with a total delivery time of $4.15 \pm 0.11 \mathrm{~min}$, excluding setup and imaging time. The CK plans estimated treatment time per fraction is $34 \pm 0.5 \mathrm{~min}$, which includes setup and imaging time of about 
5 minutes. The CyberKnife system takes two orthogonal kilo-voltage X-rays during the treatments at a selected time interval which affects the treatment time.

\section{Limitation of the study and Halcyon ${ }^{\mathrm{TM}} 2.0$ plan delivery}

CK sequential planning is well-known planner experience dependent. The limited number of plans in the study may increase the variation of dosimetric results. It is also reflected from the relatively large standard deviation bars for CK in Figure 1. The newly introduced CK VOLO optimizer may generate plans with less user dependent. In the future, it may worth a further evaluation and comparison for the CK plans with MLC option.

Plans are compared only dosimetrically. However, patient positioning and motion management are still potentially problematic with the Halcyon ${ }^{\mathrm{TM}} 2.0$ system since currently there is no intrafraction monitoring, as with the Varian EDGE/TrueBeam line, and the couch has only three degrees of freedom. The couch has no rotational corrections, and if the position needs to be corrected rotationally, this can be done manually only in the yaw direction in the room. These shortcomings make it especially critical to establish practical pretreatment prep protocol and to strongly advise the patients to follow the protocol. The patients are to follow the same protocol before the CT sim and each actual treatment delivery, ensuring the ideal patient setup during the actual treatment delivery.

The Halcyon ${ }^{\mathrm{TM}} 2.0$ requires daily imaging before each treatment due to lack of light field and the optical distance indicator. Therefore, CBCT or portal images have to be taken before each treatment. Cai et al. showed that Halcyon 2.0 kV-CBCT with iterative reconstruction satisfies requirements for clinical use [25]. The same study also reported CBCT acquisition time at Halcyon 2.0 is improved and faster compared to the $\mathrm{C}$-arm linac systems. That gives the option of taking a fast $\mathrm{kV}$-CBCT before each or selected beams to verify fiducial or anatomy matching and apply the shifts immediately before the treatment beam. Even though there is no intrafraction tracking with Halcyon 2.0, CBCT provides three-dimensional data of the target and critical organs. The Halcyon 2.0 system at the FROS clinic has Pelvis Fast and Pelvis Large Fast protocols with a scan time of $21.2 \mathrm{~s}$ and $25 \mathrm{~s}$, respectively. Both protocols have $2.0 \mathrm{~mm}$ thickness. We expect Varian will make the intrafraction tracking with Halcyon available in the near future, which will provide additional safeguarding for the hypo-fractionated SBRT delivery. Until then, an extra CBCT before each arc delivery is warranted.

\section{Conclusions}

This study investigates the potential of SBRT prostate treatment on a Halcyon ${ }^{\mathrm{TM}} 2.0$ unit. The results show that all the re-plans on a HalcyonTM 2.0 meet the criteria of the RTOG 0938 protocol. Dosimetrically, the Halcyon $^{\mathrm{TM}} 2.0$ is capable of generating acceptable SBRT prostate plans based on the RTOG 0938 protocol goals and constraints.

\section{Additional Information \\ Disclosures}

Human subjects: Consent was obtained by all participants in this study. Animal subjects: All authors have confirmed that this study did not involve animal subjects or tissue. Conflicts of interest: In compliance with the ICMJE uniform disclosure form, all authors declare the following: Payment/services info: All authors have declared that no financial support was received from any organization for the submitted work. Financial relationships: All authors have declared that they have no financial relationships at present or within the previous three years with any organizations that might have an interest in the submitted work. Other relationships: All authors have declared that there are no other relationships or activities that could appear to have influenced the submitted work.

\section{References}

1. Benedict SH, Yenice KM, Followill D, et al.: Stereotactic body radiation therapy: the report of AAPM Task Group 101. Med Phys. 2010, 37:4078-4101. 10.1118/1.3438081

2. Zelefsky MJ, Chan H, Hunt M, Yamada Y, Shippy AM, Amols H: Long-term outcome of high dose intensity modulated radiation therapy for patients with clinically localized prostate cancer. J Urol. 2006, 176:14151419. 10.1016/j.juro.2006.06.002

3. Dașu A: Is the $\alpha / \beta$ value for prostate tumours low enough to be safely used in clinical trials? . Clin Oncol. 2007, 19:289-301. 10.1016/j.clon.2007.02.007

4. King CR, Brooks JD, Gill H, Pawlicki T, Cotrutz C, Presti JC Jr: Stereotactic body radiotherapy for localized prostate cancer: interim results of a prospective phase II clinical trial. Int J Radiat Oncol Biol Phys. 2009, 73:1043-1048. 10.1016/j.ijrobp.2008.05.059

5. Katz AJ: CyberKnife radiosurgery for prostate cancer . Technol Cancer Res Treat. 2010, 9:463-472. $10.1177 / 153303461000900504$

6. Freeman DE, King CR: Stereotactic body radiotherapy for low-risk prostate cancer: five-year outcomes . Radiat Oncol. 2011, 6:3. 10.1186/1748-717X-6-3

7. Katz AJ, Santoro M, Diblasio F, Ashley R: Stereotactic body radiotherapy for localized prostate cancer: 
disease control and quality of life at 6 years. Radiat Oncol. 2013, 8:118. 10.1186/1748-717X-8-118

8. Katz A: Stereotactic body radiotherapy for low-risk prostate cancer: a ten-year analysis . Cureus. 2017, 9:e1668. 10.7759/cureus.1668

9. Kishan AU, Dang A, Katz AJ, et al.: Long-term outcomes of stereotactic body radiotherapy for low-risk and intermediate-risk prostate cancer. JAMA Netw Open. 2019, 2:188006. 10.1001/jamanetworkopen.2018.8006

10. Antypas C, Pantelis E: Performance evaluation of a CyberKnife ${ }^{\circledR} \mathrm{G} 4$ image-guided robotic stereotactic radiosurgery system. Phys Med Biol. 2008, 53:4697-4718. 10.1088/0031-9155/53/17/016

11. King CR, Lehmann J, Adler JR, Hai J: CyberKnife radiotherapy for localized prostate cancer: rationale and technical feasibility. Technol Cancer Res Treat. 2003, 2:25-29. 10.1177/153303460300200104

12. Kilby W, Dooley JR, Kuduvalli G, Sayeh S, Maurer CR Jr: The CyberKnife ${ }^{\circledR}$ robotic radiosurgery system in 2010. Technol Cancer Res Treat. 2010, 9:433-452. 10.1177/153303461000900502

13. Kim MM, Bollinger D, Kennedy C, et al.: Dosimetric characterization of the dual layer MLC system for an ORing linear accelerator. Technol Cancer Res Treat. 2019, 18:153303381988364. 10.1177/1533033819883641

14. Petroccia HM, Malajovich I, Barsky AR, et al.: Spine SBRT with HalcyonTM: plan quality, modulation complexity, delivery accuracy, and speed. Front Oncol. 2019, 9:319. 10.3389/fonc.2019.00319

15. Lukka H, Bahary J, Lawton C, et al.: A randomized phase II trial of hypofractionated radiotherapy for favorable risk prostate cancer - RTOG CCOP Study. NRG Oncol. 2015,

16. Paddick I: A simple scoring ratio to index the conformity of radiosurgical treatment plans: technical note . J Neurosurg. 2000, 93:219-222. 10.3171/jns.2000.93.supplement_3.0219

17. Feuvret L, Noël G, Mazeron J-J, Bey P: Conformity index: a review. Int J Radiat Oncol Biol Phys. 2006, 64:333-342. 10.1016/j.ijrobp.2005.09.028

18. Shaw E, Kline R, Gillin M, Souhami L, Hirschfeld A, Dinapoli R, Martin L: Radiation therapy oncology group: radiosurgery quality assurance guidelines. Int J Radiat Oncol Biol Phys. 1993, 27:1231-1239. 10.1016/03603016(93)90548-A

19. Zhang P, Happersett L, Hunt M, Jackson A, Zelefsky M, Mageras G: Volumetric modulated arc therapy: planning and evaluation for prostate cancer cases. Int J Radiat Oncol Biol Phys. 2010, 76:1456-1462. 10.1016/j.ijrobp.2009.03.033

20. Holt A, van Vliet-Vroegindeweij C, Mans A, Belderbos JS, Damen EMF: Volumetric-modulated arc therapy for stereotactic body radiotherapy of lung tumors: a comparison with intensity-modulated radiotherapy techniques. Int J Radiat Oncol Biol Phys. 2011, 81:1560-1567. 10.1016/j.ijrobp.2010.09.014

21. Lin Y-W, Lin K-H, Ho H-W, Lin HM, Lin LC, Lee SP, Chui CS: Treatment plan comparison between stereotactic body radiation therapy techniques for prostate cancer: non-isocentric CyberKnife versus isocentric RapidArc. Phys Med. 2014, 30:654-661. 10.1016/j.ejmp.2014.03.008

22. Hegazy M, Mahmood R, Nobah A, Moftah B, Alzorkany F: Stereotactic radiotherapy for localized prostate cancer, which is better Cyberknife or RapidArc?. J Pros Canc. 2016, 1:5.

23. MacDougall ND, Dean C, Muirhead R: Stereotactic body radiotherapy in prostate cancer: is Rapidarc a better solution than Cyberknife?. Clin Oncol. 2014, 26:4-9. 10.1016/j.clon.2013.08.008

24. Pinter C, Lasso A, Wang A, Jaffray D, Fichtinger G: SlicerRT: radiation therapy research toolkit for 3D Slicer . Med Phys. 2012, 39:6332-6338. 10.1118/1.4754659

25. Cai B, Laugeman E, Mazur TR, et al.: Characterization of a prototype rapid kilovoltage $\mathrm{x}$-ray image guidance system designed for a ring shape radiation therapy unit. Med Phys. 2019, 46:1355-1370. 10.1002/mp.13396 